\title{
Coping after recurrent miscarriage: uncertainty and bracing for the worst
}

\author{
Henrietta D L Ockhuijsen, ${ }^{1}$ Jacky Boivin, ${ }^{2}$ Agnes van den Hoogen, ${ }^{3}$ \\ Nickolas S Macklon ${ }^{4}$
}

${ }^{1} \mathrm{PhD}$ Student, Department of Reproductive Medicine and Gynaecology, University Medical Centre Utrecht, Utrecht, The Netherlands

2Professor, School of Psychology, Cardiff University, Cardiff, UK

${ }^{3}$ Nurse Researcher, Department of Neonatology, Wilhelmina Children's Hospital and University Medical Centre Utrecht, Utrecht, The Netherlands

${ }^{4}$ Professor of Obstetrics and Gynaecology, Human Development and Health, University of Southampton, Southampton, UK

\section{Correspondence to} Ms Henrietta D L Ockhuijsen, Department of Reproductive Medicine and Gynaecology, University Medical Centre Utrecht, PO Box 85500, Utrecht 3508 GA, The Netherlands; h.d.I ockhuysen@umcutrecht.nl

Received 28 February 2012 Revised 8 October 2012 Accepted 10 October 2012 Published Online First

17 January 2013
To cite: Ockhuiijsen HDL, Boivin J, van den Hoogen A, et al. J Fam Plann Reprod Health Care 2013;39:250-256

\begin{abstract}
Background The aim of this study was to understand how women with single or recurrent miscarriages cope during the waiting periods after miscarriage - waiting for pregnancy or waiting for pregnancy confirmation - and to investigate their perception of a 'positive reappraisal' coping intervention designed for these waiting periods. Positive reappraisal is a cognitive strategy to change the meaning of a situation, specifically reinterpreting the situation in a more positive way.
\end{abstract}

Methods A qualitative methodology was used. Data were obtained from two focus groups comprising nine women with one or more miscarriages.

Results Two core categories, 'uncertainty' and 'bracing', were highlighted during the waiting period for confirmation of an ongoing pregnancy. Women who had experienced a single miscarriage appraised this waiting period as benign and used distraction and coping by social support. Women with recurrent miscarriages could not confidently appraise the waiting period as one that would bring hope or joy and used bracing for the worst as their coping strategy to manage this ambivalence. With this strategy, women tried to control their current emotions, and looked into the future to try to minimise their distress if a further miscarriage occurred. Although all women thought that a 'positive reappraisal' coping intervention would be practical and applicable during waiting periods, only women with recurrent miscarriages actually wanted to use such an intervention.

Conclusions Coping interventions targeting reappraisal of the waiting period stressor situation could help women to cope as they wait for a subsequent pregnancy to be confirmed as ongoing. Coping interventions may need to be tailored, but before any strategy is introduced, further study is needed to identify the most appropriate approach.

\section{BACKGROUND}

More than one in 10 pregnancies will end in a miscarriage and this risk increases with age. Further, between 1\% and $3 \%$ of women will suffer recurrent miscarriages, with an underlying cause found in fewer than 50\% of such couples. Miscarriage is a cause of psychosocial distress, as for many women it means more than the loss of a pregnancy. It represents the feeling of a lost baby, a lost future child and a lost motherhood. Miscarriages also cause physical trauma, sudden pain, blood loss and unexpected admission to hospital. ${ }^{12}$ From their practice, health care workers know that women who have suffered miscarriages potentially experience two subsequent waiting periods: the period between renewed attempts to conceive and conception (waiting for conception), and the period between conception and confirmation that the pregnancy is ongoing (waiting for ongoing pregnancy). Waiting is associated with a build-up of anxiety and stress, which starts because of the uncertainty in timing of the conception and the ongoing pregnancy, but eventually also includes anticipatory anxiety about the outcome, either pregnancy loss or live birth. ${ }^{3}$

Women who have experienced miscarriages may benefit from psychosocial support and counselling during these waiting periods. ${ }^{1}$ Several studies have investigated the influence of psychosocial interventions in women who have experienced miscarriage. ${ }^{4-8}$ Most have focused on the period immediately following miscarriage. $^{5-8}$ There is less information relating to support during the first trimester of a subsequent pregnancy. ${ }^{4}$ Interventions such as counselling sessions with nurses, ${ }^{7}$ psychological counselling ${ }^{6}$ 
or a weekly ultrasound $\operatorname{scan}^{4}$ have been shown to reduce anxiety and depressive symptoms. Not all patients use or have access to these interventions and many request adjunct interventions that could be used in addition to such interventions, or as alternatives if they are not readily available. ${ }^{8}$

Lancastle and Boivin ${ }^{9}$ recently developed a short self-help Positive Reappraisal Coping Intervention (PRCI) for use during medical waiting periods. It consists of daily reading of 10 positive statements encouraging the use of 'positive reappraisal coping'. This is a cognitive strategy to change the meaning of a situation, in particular, reinterpreting the situation as it stands in a more positive way. ${ }^{9}$ The PRCI is based on the stress theory of Lazarus and Folkman. ${ }^{10}$ According to this theory, emotional processes are dependent on actual expectations about the significance and outcome of a specific situation and how people cope following these appraisals. ${ }^{10}$ People use a variety of coping strategies to manage stressful events. ${ }^{10}$ Problem-focused coping strategies are aimed at confronting and seeking solutions to a situation, while emotion-focused coping strategies focus on ameliorating the associated level of emotional distress. Meaning-based strategies as used in the PRCI (e.g. deriving benefit from adversity or focusing on the positive) are future-orientated strategies that have been shown to be effective where sustained coping efforts are required when a stressor situation is uncontrollable and its outcome unpredictable. ${ }^{11}{ }^{12}$ This is the case in medical waiting periods such as those after experiencing miscarriages. Another future-orientated coping strategy is 'bracing'. Bracing occurs as an attempt to anticipate unpleasant surprises, presumably to avoid disappointment. ${ }^{13-16}$

The PRCI was originally developed for the waiting period after embryo transfer in infertility treatment. It consists of a small card (Figure 1) that contains 10 positive reappraisal statements, together with a leaflet with detailed information about the coping techniques. Women are asked to read the PRCI at least twice a day, in the morning and evening, and at any other time that they feel the need to do so. Women have to read the statements and think about how each statement applies to them personally. As conception or confirmation of ongoing pregnancy after a miscarriage often involve a sustained period of waiting, we felt that the PRCI could potentially also be a useful adjunct intervention for women in this patient group. To adapt and further develop the PRCI for women with miscarriages, the UK Medical Research Council medical framework for developing complex interventions was used. ${ }^{16}$

This article presents the results of the first phase of the study, which was to describe the coping strategies of women after miscarriage to determine whether the PRCI intervention could also be applied to this population. To model the intervention, we used a qualitative

\section{During this experience I will:}

Try to do something that makes me feel good

See things positively

Look on the bright side of things

Make the best of the situation

Focus on what is important in life

Focus on the positive aspects of the situation

Find something good in what is happening

Try to do something meaningful

Focus on the benefits and not just the difficulties

Learn from the experience

Figure 1 Positive Reappraisal Coping Intervention. ${ }^{9}$ C 2008 Cardiff University. Figure reproduced with the kind permission of the authors and Cardiff University.

study design involving two focus groups as recommended for implementation of interventions in novel contexts. ${ }^{17}$ In the focus groups we aimed to explore the experience, coping styles and strategies of women during the waiting period for ongoing pregnancy.

\section{METHODS}

\section{Participants}

Women attending an Early Pregnancy Unit and/or Recurrent Miscarriage Clinic managed by nurses and doctors at the University Medical Centre Utrecht in The Netherlands were invited to participate in this study. Twenty-five participants, all Dutch-speaking, were approached by telephone and 14 agreed to participate. The main reason for declining was reluctance to talk about this subject in a group setting. The potential participants were assigned to focus groups based on the number of miscarriages that they had experienced. One focus group comprised women who had experienced a single miscarriage, were currently more than 12 weeks pregnant and were waiting for confirmation of ongoing pregnancy (SM group). Of the seven women invited to this group, one did not attend and two cancelled due to illness. The final sample size in the SM group was therefore four women. The second focus group comprised women with recurrent miscarriages of whom two were 
over 12 weeks pregnant (RM group). Of the seven women invited to this group, two women did not attend, one because of emotional problems and one for practical reasons. The final sample size in the RM group was therefore five women. The focus groups took place in a meeting room outside the clinic. Demographic characteristics of the 14 women who agreed to participate are given in Table 1.

\section{Data collection}

An interviewer, note-taker and the researcher, all female, were present during the two focus group sessions. The interviewer had a degree in health science and was experienced in leading focus groups. Notes were taken by a psychologist and the researcher was present to observe.

Data collection took place by semi-structured interview to address issues relevant to miscarriages. The interview schedule broadly followed questions about experiences and coping strategies around waiting for conception and waiting for ongoing pregnancy. During the focus group session the PRCI was provided and explained, but not used by the women. Questions were asked about the perceptions of the feasibility and acceptability of the PRCI. The central questions for the focus groups were: "How have women with single or recurrent miscarriages experienced and coped during the waiting periods after miscarriage?" and "What is their opinion about the usefulness of the PRCI?" As most of the women in the RM group were not yet pregnant, their experiences about waiting for an ongoing pregnancy related to past pregnancies that had miscarried. Prompts were developed to ensure that women covered specific categories. The session continued until no new data were gathered, that is, until the data were saturated. The focus groups each lasted $2^{1 / 2}$ hours with a break of 15 minutes.

\section{Data analysis}

The interviews were tape-recorded and transcribed in full. The method of grounded theory was used to analyse the data of focus groups. ${ }^{18}$ In this method, three levels of the coding are used: open, axial and selective coding. ${ }^{19}$ The interviews were organised and analysed by thematic analysis assisted by the software programme MAXQDA 10 ${ }^{\mathrm{TM}}$ (VERBI Software $\mathrm{GmbH}$, Marburg, Germany). To validate the accuracy of the findings, virtual repeatability was used. ${ }^{20}$ This was made possible by transcribing the interviews, making field notes and using peer review and peer debriefing with two colleagues proficient in qualitative research to ensure repeatability of findings. One colleague reanalysed the raw data with subsequent consensus discussions with the researcher about emerging categories. Member checking took place during the interviews by asking the participants whether the summaries were a true reflection of their reality.

Illustrative quotations were edited for ease of reading and relevance using the following notation system: '...' refers to omission of some part of the quotation because it is irrelevant to the argument. Where necessary for clarity, additional text (indicated by square brackets) has been included for ease of reading and comprehensibility. Each quotation is followed by a fictitious name (see Table 1), true age and number of miscarriages experienced by the respondent. Translation of the quotations for the purposes of this article was by the researcher and a native English speaker carried out back-translations to verify their accuracy.

\section{Ethics approval}

Permission to conduct the study was obtained from the Ethical Committee of the University of Utrecht, Utrecht, The Netherlands.

Table 1 Demographic characteristics of participants in the single miscarriage and recurrent miscarriage focus groups

\begin{tabular}{llllll}
\hline Name & Age (years) & Children $(\boldsymbol{n})$ & Miscarriages $(\boldsymbol{n})$ & Pregnant & Attendance at focus group \\
\hline $\begin{array}{lllll}\text { Single miscarriage (SM) } \\
\text { Anna }\end{array}$ & 36 & 0 & 1 & Yes & Did not attend due to illness \\
Bea & 29 & 0 & 1 & Yes & Did not attend due to illness \\
Cecile & 29 & 0 & 1 & Yes & Yes, conception time 3 months, 15 weeks pregnant \\
Diana & 29 & 0 & 1 & Yes & Yes, conception time 12 months, 26 weeks pregnant \\
Eva & 28 & 4 & 1 & Yes & Did not attend, unspecified \\
Freya & 34 & 0 & 1 & Yes & Yes, conception time 2 months, 21 weeks pregnant \\
Gloria & 31 & 2 & 1 & Yes & Yes, conception time 6 months, 26 weeks pregnant \\
Recurrent miscarriage (RM) & & & & \\
Helen & 34 & 0 & 3 & No & Yes, 5 months after last miscarriage \\
Irene & 38 & 1 & 3 & No & Yes, 3 months after last miscarriage \\
Julia & 30 & 0 & 3 & Yes & Did not attend due to emotional problems \\
Karen & 33 & 1 & 3 & Yes & Yes, conception time 2 months, 16 weeks pregnant \\
Lucy & 34 & 1 & 4 & No & Yes, 5 months after last miscarriage \\
Maria & 29 & 1 & 3 & No & Did not attend for practical reasons \\
Nancy & 38 & 0 & 6 & No & Yes, 5 months after miscarriage \\
\hline
\end{tabular}




\section{RESULTS}

The results of open coding are presented, followed by the axial and selective coding.

\section{Open-coding categories}

Open coding produced the following four categories: experiences, appraisal, coping and PRCI. The categories and their subcategories as indicated by women in the two focus groups are shown in Table 2.

Waiting period for conception

Experiences

Women with the experience of SM or RM reported that the waiting period for ongoing pregnancy was not as stressful as the time immediately after the miscarriage. All women judged the latter period as the worst time. Both groups had feelings of grief because of the loss of a future baby and felt in need of more support during this period.

"What I found the most difficult were the first weeks after the miscarriage. When I look back, this was the hardest time. You are disappointed and you have physical problems. If I have to face that again I will use all the help there is." [Helen, 34-3].

Women who had experienced one miscarriage had the feeling that their miscarriage was just bad luck. They still had hope for the future.

"It is a bit of a false start feeling. You are positioned in the starting blocks and you think ... but we can have a new opportunity. You have no reason for worrying too much.” [Freya, 34-1]

Women with RM had fears for the future and some women were afraid of never having another pregnancy or children of their own.

"What I find difficult is the thought that I might never have children and no family but what is very bad is that what I have now apparently is not enough. I am so busy wondering whether I will ever have children." [Helen, 34-3]

Table 2 Categories and subcategories emerging from open coding in the single miscarriage and recurrent miscarriage focus groups

\begin{tabular}{|c|c|c|}
\hline Category & Single miscarriage group & $\begin{array}{l}\text { Recurrent miscarriage } \\
\text { group }\end{array}$ \\
\hline \multicolumn{3}{|c|}{ Waiting period for conception } \\
\hline Experiences & $\begin{array}{l}\text { Time after miscarriage } \\
\text { Hope for the future }\end{array}$ & $\begin{array}{l}\text { Time after miscarriage } \\
\text { Fear for the future }\end{array}$ \\
\hline \multicolumn{3}{|c|}{ Waiting period for ongoing pregnancy } \\
\hline Appraisal & Challenge & Uncertainty \\
\hline Coping & $\begin{array}{l}\text { Emotion-focused coping } \\
\text { Informing a broader social } \\
\text { network }\end{array}$ & $\begin{array}{l}\text { Emotion-focused coping } \\
\text { Controlling } \\
\text { Bracing } \\
\text { Informing a specific social } \\
\text { network }\end{array}$ \\
\hline $\mathrm{PRCl}$ & $\begin{array}{l}\text { Practical and applicable } \\
\text { No need to use it }\end{array}$ & $\begin{array}{l}\text { Practical and applicable } \\
\text { Need to use it }\end{array}$ \\
\hline
\end{tabular}

$\mathrm{PRCl}$, Positive Reappraisal Coping Intervention.
Waiting period for ongoing pregnancy

Appraisal of the waiting period for ongoing pregnancy

Women with SM or RM differed in the way they appraised the waiting period for ongoing pregnancy. Women in the SM group were a little uncertain during the waiting period but they still mainly appraised the waiting period as benign or a (positive) challenge rather than a threat with potential for harm.

"A girlfriend has had four miscarriages. That is a completely different story. Her experiences were quite different. When I compare myself to her, I do not have strong feelings, almost nothing.” [Gloria, 31-1]

Women in the RM group did not know or were unable to appraise the waiting period: is it benign, a challenge, threat or harm? As a result women in the RM group were very uncertain about how they should regard or feel about the pregnancy.

"I noticed that two things were present in a subsequent pregnancy. You are reminded of your loss or more losses and that causes extra sadness and I had the feeling that I did not want to lose this child, and on the other hand I wanted to love this child. That makes you very insecure and afraid." [Julia, 34-3]

The length of time of the uncertainty depended on the previous experiences. Some women were uncertain for the first weeks while others were uncertain for up to 20 weeks.

"I had an ultrasound at 7 weeks and then I heard the heartbeat. Only this does not give certainty because the last pregnancy ended at 7 weeks. It [uncertainty] has actually lasted up to 20 weeks before I thought yes, it is now really well." [Gloria, 31-1]

Women with RM declared that the uncertainty grew after every new miscarriage.

"We were actually still not quite accustomed to the idea, and then it went wrong. That is an entirely different approach than when one is very focused on the pregnancy. The second time that it goes wrong .... that happens ... but after the second time it is becoming more precarious because then you do not know the cause and I would also not know how you might affect it.” [Lucy, 34-4]

Coping during the waiting period for ongoing pregnancy

The coping strategies the two groups used the most were emotion-focused strategies like avoidance, seeking social support, positive reappraisal and distraction. The main difference between women with SM or $\mathrm{RM}$ was the reason for using the coping strategies.

Women with SM made a point of trying to cope in a different way than during their first pregnancy. For instance, one woman avoided sports during the first pregnancy because she was afraid that it was bad for the baby, however in the second pregnancy she ran a marathon. Some women avoided seeking information on the internet or avoided thinking too much about 
the baby. Others searched for social support by informing a broader social network sooner about the pregnancy to anticipate a need for support in case of a possible new miscarriage.

"I told family and friends in both pregnancies, but earlier in the second because they already knew of the previous miscarriage. Yes it helps to talk about it because when it goes wrong again you can have more support from those people." [Cecile, 29-1]

Women with RM primarily used the coping strategy of bracing for the future. These women tried to control their emotions and future emotions as much as possible to prepare for the worst outcome. They anticipated negative feelings that could be caused by a new miscarriage in the future. For instance, they avoided thinking and daydreaming about the baby.

"You notice the thoughts of no planning ahead, no dreaming ... I wanted to be happy really ... I had every reason to but ever since that [miscarriage] you are feeling cautious." [Lucy, 34-4]

Women in both groups distracted themselves by going on a holiday or meeting a friend, but it was not always effective. The thought of a possible new miscarriage was frequently on their minds.

"You try distraction but it does not always work. You try it but you always carry it [the miscarriage] with you, so it is more about killing the time." [Karen, 33-2]

Women with RM reported trying to control their social support as much as possible by informing a smaller group of people in case of a subsequent conception. They informed only those people who really could give good support.

"The more often I became pregnant the fewer people I told [of the pregnancy]. The first time I told everyone who was willing to hear it. The last time I only told a cousin.” [Nancy, 38-6]

\section{Perceptions of PRCI}

The SM and RM groups thought that the PRCI could be practical and applicable. However, most women with SM did not feel the need to use an intervention whereas most women with RM did. One woman with repeated miscarriage stated:

"Yes, I would use it, you want to do something. There is nothing else I can do, and now there is something. I think for that reason it can be very helpful." [Helen, 34-3]

Women with a single miscarriage could imagine that the PRCI would be useful to other women with more negative miscarriage experiences.

"Yes if you really have all the negative thoughts and you do not know how to handle it then I think it is an excellent tool ... you focus on the positive things. But for me personally, I would not use it." [Bea, 29-1]
Both groups suggested that perhaps the card would be more useful if women additionally kept a diary.

"I don't think it is difficult to use. But it might help more if you write something in the morning about the statements and then in the evening you could evaluate." [Cecil, 29-1]

\section{Axial and selective coding}

The two core categories 'uncertainly' and 'bracing' emerged out of the data from open coding. These core categories explained the differences between SM and RM in coping and have an association with the open coding categories found.

Uncertainty

Waiting for ongoing pregnancy is an event that women with SM experienced and coped with differently to women with RM. The more miscarriages a woman had experienced, the more difficult it became in a subsequent pregnancy to confidently appraise the waiting period for ongoing pregnancy. Women with SM still experienced a new pregnancy as benign or a (positive) challenge while women with RM became more uncertain in a subsequent pregnancy. This uncertainty expanded with the increase in the number of miscarriages.

Bracing

Both groups used emotion-focused coping strategies aimed at regulating emotions they were experiencing, but women with RM used the coping strategy to control or brace against their current emotions and the possible future emotions arising from a negative outcome. SM women just used them to cope in a different way than during their first pregnancy.

\section{Relationship between uncertainty and bracing}

Uncertainty appears when women do not know how to appraise the 'waiting for ongoing pregnancy' period. They do not know if the waiting period will be a challenge, threat or harm because the outcome becomes more unpredictable the greater the number of miscarriages. The more miscarriages women have experienced the more the uncertainty grows. It grows because women have less faith that they will ever have an ongoing pregnancy. Women brace as a coping strategy to deal with this uncertainty. Bracing is an attempt to control the emotions and future emotions as much as possible, and to prepare for the worst outcome.

\section{DISCUSSION}

This qualitative study was aimed at exploring the coping strategies in women with single and recurrent miscarriage. We also examined whether a PRCI was perceived as useful for this population. The results show that two core categories, 'uncertainty' and 'bracing', differed between women with RM or SM. The more miscarriages women had experienced, the more likely that bracing was adopted as the core 
coping strategy to deal with increasing uncertainty about a current or eventual pregnancy. Women thought that coping interventions during the waiting period could be useful and that these could include positive reappraisal tools such as PRCI or other cognitive or psycho-educational interventions. ${ }^{12}$

All women thought that the PRCI could be practical and applicable but most women with SM did not want to use this or any other intervention, in contrast with women with RM who did. This asymmetry may be due to differences between groups in appraisal of the situation. Women with SM felt that the first miscarriage was bad luck, and expected the present pregnancy to continue, lessening the need for additional support. In contrast, women with RM clearly lacked confidence about future outcomes, with perceptions and coping orientated toward potential failure (i.e. bracing for the worst). Another explanation for the difference can be found in the Common Sense Model. ${ }^{21}$ This model proposes that people make mental representations of their illness using different sources of information, for instance from memory, social environment and somatic information. Mental representations may change with the increasing number of miscarriages. In this cognitive context, women with RM may benefit from coping strategies targeting reappraisal, such as PRCI. These findings support conclusions from a recent survey on the modes of support likely to be valued by women with RM. ${ }^{22}$

The use of the coping strategy 'brace for the worst', by which women try to control their emotions and future emotions as much as possible, has not previously been described among women with RM. However, in qualitative studies, similar behaviour has been reported such as "holding back emotions" 23 and "emotional cushioning". ${ }^{24}$ In a longitudinal, qualitative study among 82 pregnant women who had experienced loss, a number of comparable coping styles were reported. ${ }^{25}$ For example, some women were hesitant to express their growing self-assurance because they were afraid to "jinx" their pregnancy and they delayed the announcement of pregnancy. The women in that study actively pursued many avenues to gain control and cope with the difficulties of their pregnancies. Kiwi ${ }^{26}$ argued that patients with recurrent miscarriages might develop a protective emotional shield during pregnancy in an attempt to reduce the pain of impending loss. Norem and Cantor $^{27}$ described emotional cushioning as a process by which individuals protect themselves against threats to self-esteem in risky situations. The reason why women with SM did not use bracing is not clear. The differences could be caused by the fact that all women in the SM group were pregnant while most women in the RM group were not. Carroll et al. ${ }^{15}$ proposed that bracing was an attempt to avoid disappointment and reflected the cognitive strategy of defensive pessimism. Women with SM still had hope for the future, seeing the first miscarriage as "bad luck", and therefore experienced the new pregnancy as benign or a challenge.

In risky situations, two strategies can be used: defensive pessimism and an optimistic strategy. ${ }^{27}$ Defensive pessimism is discounting of past successes and the lowering of expectations prior to entering a situation. In the optimistic strategy, the expectations are high at the outset with post hoc restructuring of the situation when the outcome is known. ${ }^{27}$ It may be that women with SM were already using the coping style of positive reappraisal to deal with the current pregnancy. This optimism may explain the lack of bracing. Clearly all these future-oriented approaches theoretically overlap with bracing (and cushioning). However, our results and those from other studies concur that the particular characteristics of waiting for an ongoing pregnancy provoke specific cognitions and emotions that women may find difficult to manage because they refer to a future unknown, unpredictable and uncontrollable outcome. As such, more research attention should be devoted to this topic in relation to miscarriage and to whether bracing (and other futureoriented coping strategies) leads to positive or negative emotions in women with SM or RM.

The uncertainty for women in the RM group increased with every new miscarriage. The relationship between the number of miscarriages and the level of anxiety in a subsequent pregnancy is unclear. Some studies find a positive relationship ${ }^{2}$ and others no relationship. ${ }^{28} 29$

In the present study, women used mainly emotionfocused coping styles to handle anxiety, which is consistent with the context. Terry and Hynes ${ }^{30}$ argue that in low-control situations the use of emotion-focused coping is more effective than problem-focused coping. In contrast to our findings, in a longitudinal study of 82 women pregnant after previous miscarriage, the dominant form of coping was problem-focused and women appraised their pregnancies as a moderate threat. $^{30}$ These differences may be explained by the timing of the assessments. Women entered that study during their 10th to 17 th week of pregnancy. Lazarus and Folkman ${ }^{10}$ reported that the longer the waiting period, the more the period was likely to be appraised as a threat. While the pregnancy is progressing, the waiting period becomes shorter. Time can be a variable that changes the coping styles.

The main weakness of the present study was the difference between the number of miscarriages and current pregnancy status, since all women in the SM group were currently pregnant compared with just one in the RM group. All women in the RM group had had a miscarriage 3 months or longer ago and they were all waiting for conception. All women in the SM group were at more than 12 weeks of gestation. A further weakness was the non-attendance and consequent reduced sample size. It is likely that non- 
attendance resulted in a less varied representation of miscarriage experience. The minimum acceptable number of focus groups and the sample size for each group in the literature is ambiguous. ${ }^{18}{ }^{32}$ Halcomb et al. $^{32}$ advise at least two focus groups of each participant type and a group size between four and 12 participants. In our study, we used two focus groups and the final sample size (four and five) was still within an acceptable range. ${ }^{31}$

In conclusion, similarities and differences were found in the experiences of women with SM or RM. Despite the limitations of this study, the two core categories, bracing and uncertainty, were found to be important for women with RM in the waiting period for ongoing pregnancy. More research is required to understand whether modulating these coping strategies might reduce stress in women who have suffered RM. However, the presented findings indicate that the coping strategies adopted by women with recurrent miscarriage as they wait for confirmation of ongoing pregnancy are likely to be amenable to a specifically designed PRCI. A randomised study to assess whether this intervention can improve coping during this stressful waiting period is currently in progress.

\section{Funding None.}

\section{Competing interests None.}

Provenance and peer review Not commissioned; externally peer reviewed.

\section{REFERENCES}

1 Lee C, Slade P. Miscarriage as a traumatic event: a review of the literature and new implications for intervention. $J$ Psychosom Res 1996;40:235-244.

2 Fertl KI, Bergner A, Beyer R, et al. Levels and effects of different forms of anxiety during pregnancy after a prior miscarriage. Eur J Obstet Gynecol Reprod Biol 2000;142:23-29.

3 Boivin J, Lancastle D. Medical waiting periods: imminence, emotions and coping. Womens Health 2010;6:59-69.

4 Clifford K, Rai R, Regan L. Future pregnancy outcome in unexplained recurrent first trimester miscarriage. Hum Reprod 1997; 12:387-389.

5 Rowsell E, Jongman G, Kilby M, et al. The psychological impact of recurrent miscarriage, and the role of counseling at a pre-pregnancy counseling clinic. J Reprod Infant Psychol 2001;19:33-45.

6 Nikcevic AV, Kuczmierczyk AR, Nicolaides KH. The influence of medical and psychological interventions on women's distress after miscarriage. J Psychosom Res 2007;3:283-290.

7 Swanson KM, Chen HT, Graham JC, et al. Resolution of depression and grief during the first year after miscarriage: a randomized controlled clinical trial of couples-focused interventions. $J$ Womens Health (Larchmt) 2009;18:1245-1257.

8 Boivin J. A review of psychosocial interventions in infertility. Soc Sci Med 2003;57:2325-2341.

9 Lancastle D, Boivin J. A feasibility study of a brief coping intervention (PRCI) for the waiting period before a pregnancy test during fertility treatment. Hum Reprod 2008;23:2299-2307.
10 Lazarus RS, Folkman S. Stress, Appraisal, and Coping. New York, NY: Springer, 1984.

11 Folkman S. Positive psychological states and coping with severe stress. Social Sci Med 1997;45:1207-1221.

12 Folkman S, Moskowitz JT. Coping: pitfalls and promise. Annu Rev Psychol 2004;55:745-774.

13 Taylor KM, Shepperd JA. Bracing for the worst: severity, testing, and feedback timing as moderators of the optimistic bias. Pers Soc Psychol Bull 1998;24:915-926.

14 Shepperd JA, Findley-Klein C, Kwavnick KD, et al. Bracing for loss. J Pers Soc Psychol 2000;78:620-634.

15 Carroll P, Sweeny K, Shepperd JA. Forsaking optimism. Rev Gen Psychol 2006;10:56-73.

16 Campbell M, Fitzpatrick R, Haines A, et al. Framework for design and evaluation of complex interventions to improve health. BMJ 2000;321:694-696.

17 Hardeman W, Sutton S, Griffin S. A causal modelling approach to the development of theory-based behavior change programmes for trial evaluation. Health Educ Res 2005;20:676-687.

18 Holloway I, Wheeler S. Qualitative Research in Nursing (2nd edn). Oxford, UK: Blackwell Publishing, 2006.

19 Creswell JW. Qualitative, Quantitative, and Mixed Methods Aapproaches (2nd edn). Thousand Oaks, CA: Sage, 2003.

20 Maso I, Smaling A. Kwalitatief onderzoek: praktijk en theorie. Amsterdam, The Netherlands: Boom, 1998.

21 Hagger MS, Orbell S. A meta-analytic review of the common sense model of illness representations. Psychol Health 2003;18:141-184.

22 Musters AM, Taminiau-Bloem EF, van den Boogaard E, et al. Supportive care for women with unexplained recurrent miscarriage: patients' perspectives. Hum Reprod 2011;26:873-877.

23 Côté-Arsenault D, Morrison-Beedy D. Women's voices reflecting changed expectations for pregnancy after perinatal loss. J Nurs Scholarsh 2001;33:239-244.

24 Côté-Arsenault D, Donata K. Emotional cushioning in pregnancy after perinatal loss. J Reprod Infant Psychol 2011;29:81-92.

25 Côté-Arsenault D, Donato KL, Earl SS. Watching \& worrying: early pregnancy after loss experiences. MCN Am J Matern Child Nur 2006;31:356-363.

26 Kiwi R. Recurrent pregnancy loss: evaluation and discussion of the causes and their management. Cleve Clin J Med 2006;73:913-921.

27 Norem JK, Cantor N. Anticipatory and post hoc cushioning strategies: optimism and defensive pessimism in "risky" situations. Cogn Ther Res 1986;10:347-362.

28 Craig M, Tata P, Regan L. Psychiatric morbidity among patients with recurrent miscarriage. J Psychosom Obstet Gynaecol 2002;23:157-164.

29 Magee PL, MacLeod AK, Tata P, et al. Psychological distress in recurrent miscarriage: the role of prospective thinking and role and goal investment. J Reprod Infant Psychol 2003;21:35-47.

30 Terry DJ, Hynes GJ. Adjustment to a low-control situation: reexamining the role of coping responses. Annu Rev Psychol 2004;55:745-774.

31 Côté-Arsenault D. Threat appraisal, coping, and emotions across pregnancy subsequent to perinatal loss. Nurs Res 2007;56:108-116.

32 Halcomb EJ, Gholizadeh L, DiGiacomo M, et al. Literature review: considerations in undertaking focus group research with culturally and linguistically diverse groups. J Clin Nurs 2007;16:1000-1011. 